\title{
Fragility analysis of existing precast industrial frames using CFRP reinforcement
}

\author{
Jian Zhu ${ }^{1,3, a}$, Pin $\operatorname{Tan}^{2, b}$ and Jianmin Jin ${ }^{2, c}$ \\ ${ }^{1}$ Department of Civil and Hydraulic Engineering, Ning Xia University, Yinchuan 750021, China. \\ ${ }^{2}$ Earthquake Engineering Research Center, Guang Zhou University, Guangzhou 510000, China. \\ ${ }^{1}$ Department of Civil Engineering, Chang An University, Xian 710064, China.

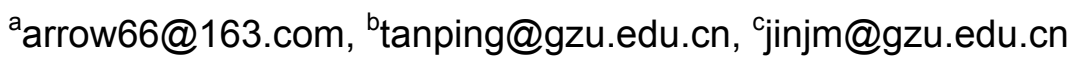

Keywords: CFRP reinforced, Existing industrial frames, Seismic fragility, Random ground motions, Nonlinear analysis.

\begin{abstract}
This study focus on derivation of such fragility curves using Carbon Fiber Reinforced Polymers (CFRP) technologies retrofitted conventional reinfored concrete (RC) factory buildings. A set of random earthquake waves which compatible with the response spectrum of major history earthquake waves in China are created. Dynamic analysis is used to compute the random sample of frames. Difference of stochastic damage scatter diagrams in two directions of buildings is considered. Seismic fragility of CFRP-reinforced industrial frames is lower than unreinforced buildings obviously, and seismic capability of frames using CFRP technologies is enhanced especially under major earthquake.
\end{abstract}

\section{Introduction}

In the last several decades, dramatic increase in the losses caused by natural catastrophes has been observed in the whole Asia-Pacific ring. Peoples are reminded once again that happen at last three major earthquakes in Eastern Asian region in the new century including Nepal Earthquake (Ms8.1) just happened in April 25, 2015. Not only the disappearing of thousands of lives but also destruction of amount of industrial and residential buildings. Especially large number of industrial buildings built between the 1990s and 2000s in Western China with economy rapid development. But many of the construction built without specific seismic provisions because of undeveloped seismic code of China in that period and later enlargement of seismic zones after Wenchuan Earthquake (Ms8.0) in 2008. So how to evaluate fragility of the industrial frames and strengthen these frames with CFRP retrofit technology for future earthquake in Western China is urgent mission.

Carbon Fiber reinforced polymers (CFRP) have a variety of advantages over other material, such as low density, high stiffness and strength, adjustable mechanical properties, resistance to corrosion, solvents and chemicals, flexible manufacturing and fast application. They have widely been used in structural repairing and seismic resistance[1-6]. In this framework, the reinforce effect of confinement introduced by the CFRP wrapping the RC columns are modeled and calculated.

Dynamic nonlinear analysis method been used in the framework. Seismic fragility analysis also introduced in the work, which is the conditional probability of a building reach or exceeding a certain performance level for a given ground motion parameter, allowing methods which correlate the damage of the building stock to these input parameters to be proposed, as shown in Eq.1.

$$
P_{i}\left(D \geq d s_{i} \mid S=S_{k}\right)=\phi\left[\frac{1}{\beta_{d s}} \operatorname{Ln}\left(\frac{S_{k}}{M_{d s}}\right)\right]
$$

Where $P_{i}$ is the probability that the damage state $d s_{i}$ will be achieved or exceeded for a ground motion severity $S_{k}, D$ and $S$ are the variables representing the damage and the severity of the input motion respectively. $\phi$ is standard normal distribution, $M_{d s}$ and $\beta_{d s}$ are the mean and standard deviation of the damage states. 


\section{System definition in industrial frames}

There are a lot of existing industrial buildings built between the 1990s and 2000s based old China seismic code in many industrial districts. The reasons which led to such large scale use are strictly related to the social and economical scenario of that time: China came into economy reform age and expand industry process. House, industrial structures were largely required and workers and construction materials were insufficiency. On the other hand, it was necessary to build rapidly and keep the cost as low as possible. Some individual buildings among those built even without any consideration of horizontal seismic loads. So nowadays they are often exposed to high levels of seismic risk. Similar structures are also occupied in other developing countries. A regular, single-storey industrial frame configuration is chosen to represent the system after widely site survey and collecting many and accurate data on the buildings characteristics in Western China. The frames are designed according to the prescriptions for loading, material, member dimensioning and detailing of the seismic design and gravity load design codes in place in former China code [7].

Based on a review of available information, a first rough classification can be done depending on the layout of main horizontal members and roof arrangement: full reticular trussed beam, so span lower than $30 \mathrm{~m}$ generally.

The structural system adopted for the precast industrial buildings could be made by straight elements, frame parts in site could be easy to manage.

The sample structures consists with general configuration of bent widths and bay widths between 4-6 $\mathrm{m}$ and 15-30 m respectively, the whole building have 24-72 meters long with 6-12 columns and 15-30 m width, It is symmetrical in plan and elevation, and rectangular reinforced concrete ring beam $(0.30 \times 0.40 \mathrm{~m})$ on the bracket of two side longitudinal columns of the building. At the same time RC column $(0.40 \times 0.60 \mathrm{~m})$ which connected ring beam as confined frame element array along the exterior side of the building at the intersect with inner confined brick wall between columns. The roof of building which height is $8-10 \mathrm{~m}$ consist in steel fibre truss, the truss depth is changed from $2.4 \mathrm{~m}$ in centre to $1.5 \mathrm{~m}$ of two sides, there are four kind of circular hollow rod being defined to using with diameter from $0.03 \mathrm{~m}$ to $0.05 \mathrm{~m}$, and thickness of bar' section is also verify from 2 to $3 \mathrm{~mm}$.

The building's wall between columns generally consist of load-bearing infill masonry walls commonly made of clay bricks in China, confined by reinforced concrete vertical columns and width and thickness of wall are changed from $0.24 \mathrm{~m}$ to $0.37 \mathrm{~m}$ according China masonry code. Columns must have four $24 \mathrm{~mm}$ and $28 \mathrm{~mm}$ diameter longitudinal reinforcements, $8 \mathrm{~mm}$ diameter stirrups must be spaced $200 \mathrm{~mm}$ in the elements. Category of available retrofitting industrial buildings is external bonded CFRP plating around each RC rectangular column.

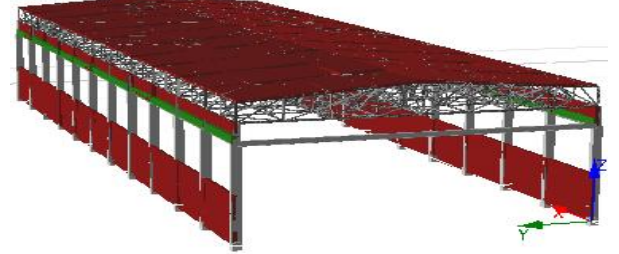

Fig.1 Finite element sample of the industrial frame

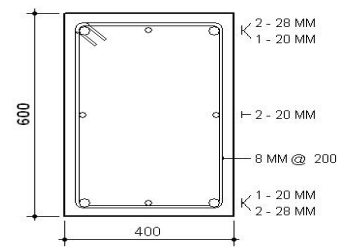

i. column section

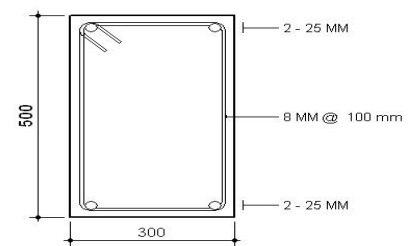

ii. beam section The gravity load scenario consists of dead load and live load. When calculating the dead load, the weight of the structural members and the masonry infill walls was included. The roof live load used was $0.5 \mathrm{kN} / \mathrm{m}^{2}$, which is typical for an industrial building. Other types of loading, such as wind and snow were not considered [8].

\section{Material and member property}

As regards the materials, the clay brick strength must at least MU15 and the mortar strength must at least M10 according to China masonry code, so typical masonry shear strength is $0.27-1 \mathrm{Mpa}$. Steel and concrete used for precast members were characterized by the actual performance available at that 
time. Bilinear stress-strain relationships with strain hardening were used for reinforced members which typical yield strength for common applications were $220 \mathrm{Mpa}$, concrete compressive strength is $25 \mathrm{Mpa}$ in considering of that many industrial frames in Western China regions have been built two decades ago. And coefficient with variation of 0.3 has been considered for steel and concrete respectively. Uniaxial nonlinear constant confinement concrete model that constant confining pressure is assumed throughout the entire stress-strain range is proposed by Madas to apply to element of concrete in this calculation [9-10]. A simplified uniaxial tri-linear CFRP model that assumes no resistance in compression is used in the research. Four model calibrating parameters are defined in order to fully describe the mechanical characteristics of the material. They are tensile strength, initial stiffness, post peak stiffness and specific weight, and the default values are $3000 \mathrm{Mpa}$, $300 \mathrm{Gpa},-500 \mathrm{Gpa}$ and $18 \mathrm{kN} / \mathrm{m}$ respectively in consideration with simplification [11].

\section{Numerical analysis}

The aim of numerical analysis is the evaluation of the seismic capacity of the reference buildings presented in the previous section, in order to determine, their seismic fragility in the evaluation the demand is identified assuming that the sample industrial buildings are placed in the area of Mianzhu region in Sichuan province of Western China, characteriazed by a PGA equal to $0.3 \mathrm{~g}$ and return period equal to 475 years according to recent China seismic classification, which are medium seismic risk areas, it is assumed that industrial buildings are located in this area affected by increase of seismic risk classification.

Typical buildings are studied by nonlinear analysis. Useful information have been obtained by analysis, made on spatial models by the computer program Seismostruct, taking into account beam-column connections geometrical eccentricities: modal shapes with vibration frequencies and axial forces of columns due to vertical loads to be combined with seismic forces.

The first three period of sample structure, which is 66 meter including 12 columns in longitudinal direction and $24 \mathrm{~m}$ in transverse direction[12], are 1.285s (longitudinal dir.), 1.094s (transverse dir.) and 1.066s (rotational), at the same time, the first three period of CFRP reinforced structure are $0.805 \mathrm{~s}, 0.696 \mathrm{~s}$ and $0.693 \mathrm{~s}$.

Ground motion characteristics have a significant effect on the fragility curves and special attention is required during the record selection phase. Considering this fact, 15 corrected stochastic artificial ground motions have been used in different intensity covering a wide range of characteristics with a magnitude range. The motions are generally recorded on soft to medium sites according to local circumstance where basis prominent period range from $0.35 \mathrm{~s}$ to $0.45 \mathrm{~s}$ according China seismic code[8].
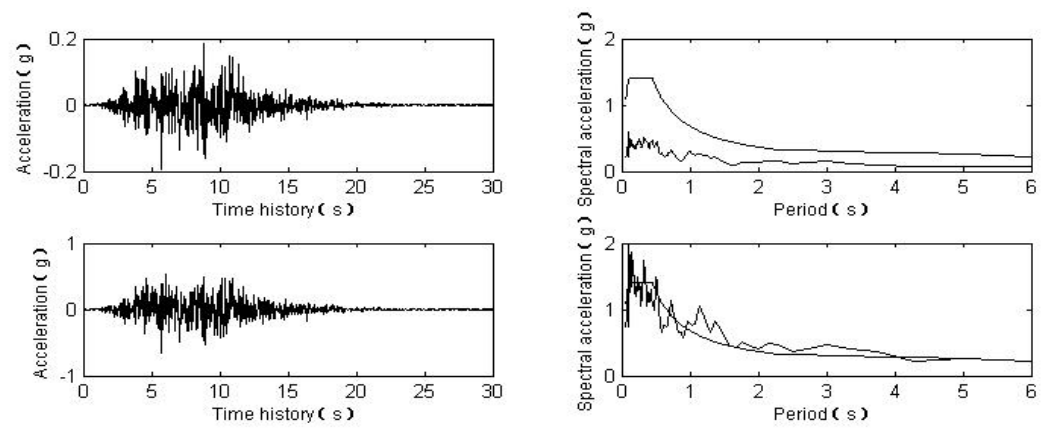

Fig.3 Random waves according response spectrum

Four limit state conditions have been taken into account: light damage, moderate damage, extensive damage and complete damage as tab.1 So if a building deforms beyond the extensive damage limit state it might not be economically benefit to repair the building because many of the industrial frames of Western China region were be set up without carefully thought of structural seismic codes of China in design[8]. 
Table 1 limit states and corresponding ISD\%

\begin{tabular}{|c|c|c|c|c|c|}
\hline Limit state & $\begin{array}{c}\text { Inter-story drift } \\
(\mathrm{mm})\end{array}$ & $\begin{array}{c}\text { Inter-story drift } \\
\text { ratio (ISD\%) }\end{array}$ & Limit state & $\begin{array}{c}\text { Inter-story drift } \\
(\mathrm{mm})\end{array}$ & $\begin{array}{c}\text { Inter-story drift ratio } \\
(\text { ISD } \%)\end{array}$ \\
\hline Light & 28.8 & 0.30 & Extensive & 115.2 & 1.20 \\
Moderate & 48 & 0.50 & Complete & 240 & 2.50 \\
\hline
\end{tabular}

\section{Fragility Curves}

Dynamic time-history analysis is used to evaluate the seismic response and to derive the seismic fragility curve. This approach is the most tedious but it is also the more direct and accurate way to assess the fragility of industrial buildings in Western China. The selected frame with or without retrofitting CFRP technology was subject to each group of the stochastic artificial records. Each group records were consisted of three stochastic ground motions.
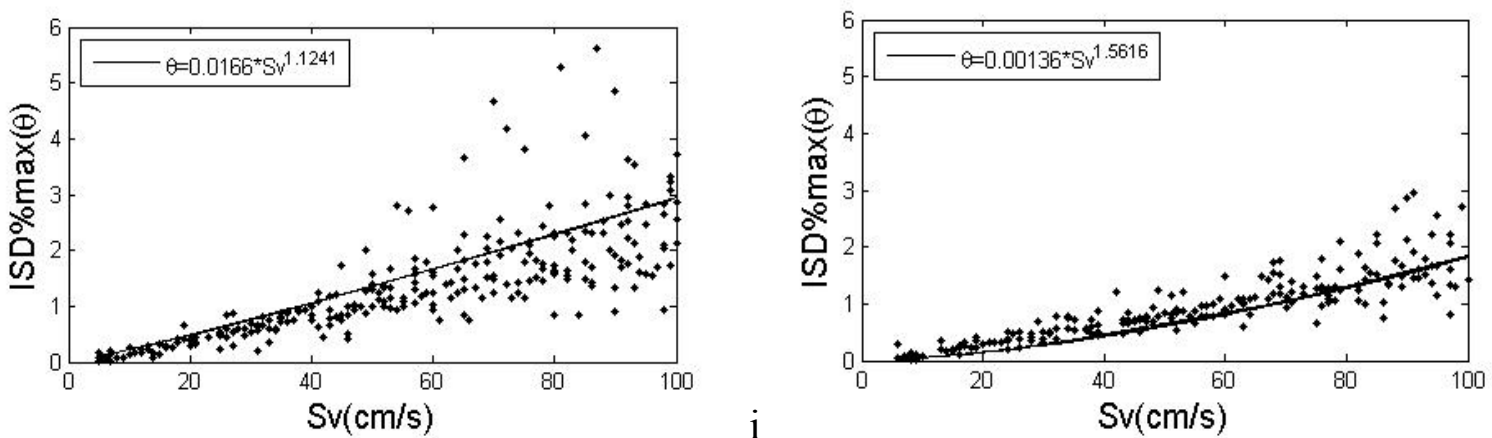

Fig.4 Stochastic damage scatter for original frames with Sv-ISD\%(i:X axis, ii:Y axis )
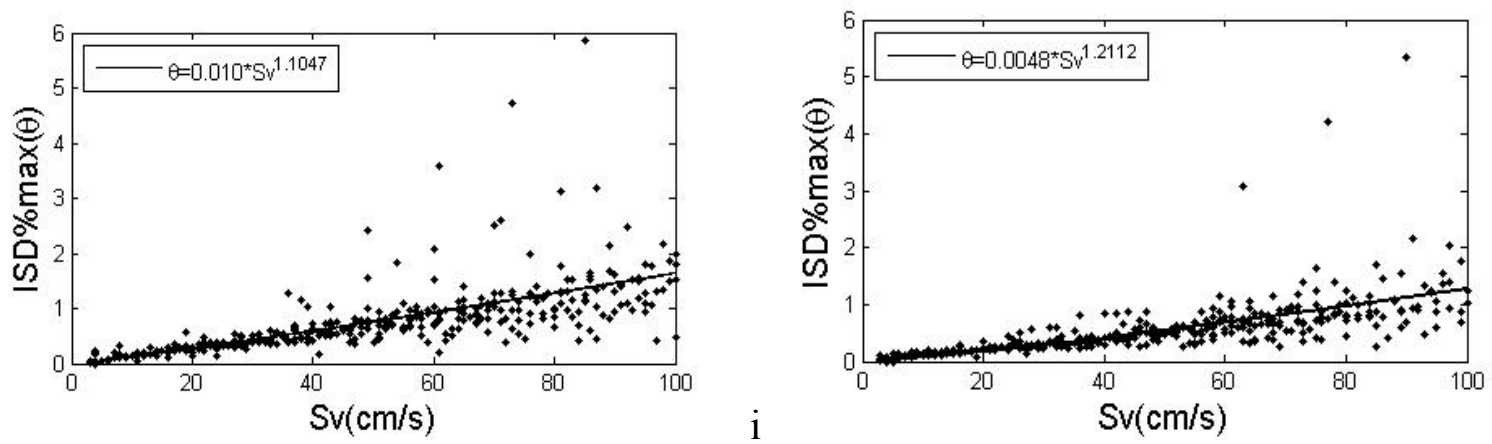

Fig.5 Stochastic damage scatter for CFRP reinforced frames with Sv-ISD\%(i:X axis, ii:Y axis )

The stochastic damage scatter diagrams were illustrated in Fig.4-Fig.5. The damage index (Y-axis) is described in terms of ISD\%. X-axis is described as spectral velocity. Scattered data depict the seismic resistant capability of each of industrial frame and corresponds to damage level. We can find that seismic capability of CFRP retrofitting frames is higher than original frames, and damage level is also lower than the un-retrofitting frames. For example, when Sv reach $100 \mathrm{~cm} / \mathrm{s}$, damage index medium value of origin model in $\mathrm{X}$ axis is equal to 0.01 , but medium value of retrofitting frame is only 0.005 , and the latter value is half of the former value. The results hint that seismic fragility of retrofitting frames is highly lower than original frames.

The best fit curve of the stochastic damage scatter data was expressed in Fig.4-5, whose statistical relationship between inter-story drift ratio $\theta$ and intensity indicators such as spectral velocity, peak ground accelerator or spectral accelerator as Eq.2:

$$
\theta=a \cdot S_{v}^{b} \quad, \theta=a \cdot p g a^{b}
$$

The scatter for each frame and each seismic intensity measure was calculated using the Eq.3:

$$
C O V=\sqrt{\frac{1}{n-2} \sum_{i=1}^{n}\left(\operatorname{In} \theta_{i}-\operatorname{In} \hat{\theta}\right)^{2}}
$$


Detail explanation in reference [13]. Similar trends were observed for all frames, as summarized in Tab.2.

Table 2 The efficiency comparison with Sv for two seismic intensity measures

\begin{tabular}{|c|c|c|c|c|c|c|}
\hline Frame & Period & Nunber of & \multicolumn{2}{c|}{ COV (\%) with original } & \multicolumn{2}{c|}{ COV (\%) with FRP } \\
\cline { 4 - 7 } Industrial frames & GM records & X axis & Y axis & X axis & Y axis \\
\cline { 2 - 7 } & 1.26 & 15 & $51.66 \%$ & $49.08 \%$ & $43.01 \%$ & $33.76 \%$ \\
\hline
\end{tabular}

Fig. 6 revealed the fragility expected contrastive results of typical used industrial frames in China with or without CFRP retrofitting in spectral velocity parameters.

The two direction seismic fragility of original sample structure was almost same especially in minor earthquake. The light and moderate damage results started when spectral velocity values equal to $10 \mathrm{~cm} / \mathrm{s}$ and $20 \mathrm{~cm} / \mathrm{s}$. The gap between moderate fragility curve and extensive fragility curve was bigger than former curves and started when spectral velocity equal to $50 \mathrm{~cm} / \mathrm{s}$. The seismic capacity of reinforced industrial buildings risen dramatically after the columns of original sample buildings were strengthen using CFRP wrapping method and extensive damage state began when spectral velocity values reach $70 \mathrm{~cm} / \mathrm{s}$. At the same time, the difference of seismic fragility in two direction was also been noticed.
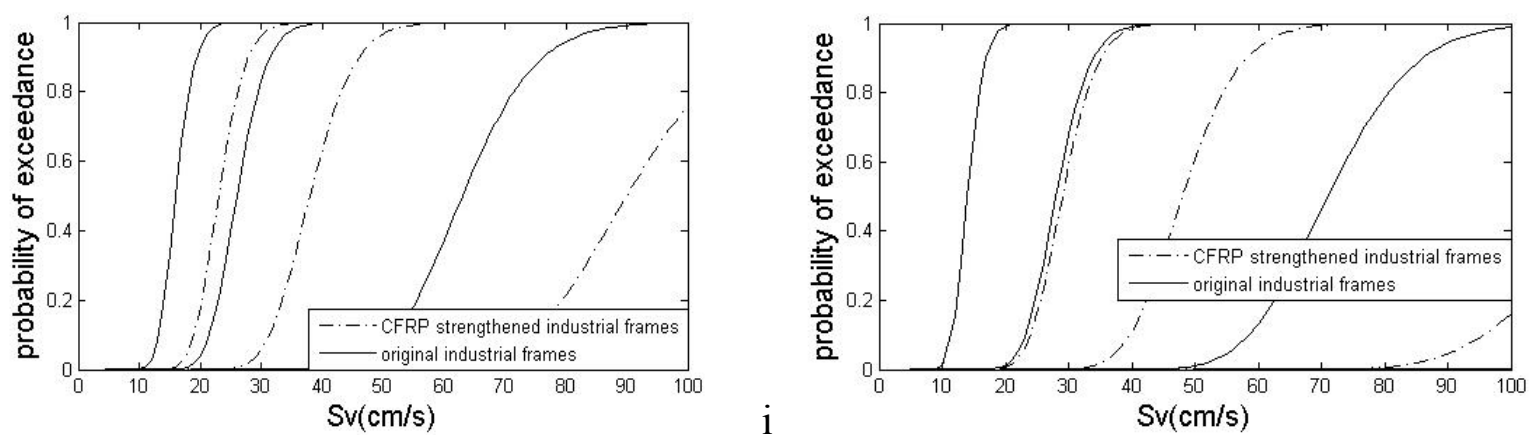

Fig.6 Seismic fragility curves of industrial frames with spectral velocity ( i: $x$ axis; ii: y axis)

\section{Conclusion}

The representative industrial buildings are chosen as researching sample for fragility curve. Dynamic nonlinear analysis was performed on the buildings, whose choice is based on a large investigation on structural typologies, details and materials. Indications as below: (1) Probability of moderate damage is much seldom when $\mathrm{Sv}<20 \mathrm{~cm} / \mathrm{s}$ under foundation stability pre-condition, in contrary probability of moderate damage was started to increasing when $\mathrm{Sv}>30 \mathrm{~cm} / \mathrm{s}$. (2) The seismic capability of longitudinal ( $\mathrm{X}$ axis) and transversal orientation ( $\mathrm{Y}$ axis) of structure is different. Probability of slight damage is coherent under minor earthquake; $\mathrm{X}$ axis of frames is more seismic fragility than Y axis under major earthquake slightly. (3) The CFRP retrofitting sample buildings had more excellent seismic capacity and decreased seismic fragility dramatically in comparison to original buildings.

\section{Acknowledgement}

This work was financially supported by the Natural Science Foundation of Ningxia (NZ13021) and National Natural Science Foundation of China (No.51468050).

\section{References}

[1] Ciro Del Vecchio, Marco Di Ludovico, Andrea Prota, Gaetano Manfredi, Analytical model and design approach for FRP strengthening of non-conforming RC corner beam-column joints, Engineering Structures, 87(2015) 8-20. 
[2] Ufuk Hancilar, Eser Çaktı, Mustafa Erdik, Guillermo E. Franco, George Deodatis, Earthquake vulnerability of school buildings: Probabilistic structural fragility analyses, Soil Dynamics and Earthquake Engineering, 67(2014) 169-178.

[3] Armin Tabandeh, Paolo Gardoni, Probabilistic capacity models and fragility estimates for RC columns retrofitted with FRP composites, Engineering Structures, 74(2014) 13-22.

[4] P.G. Asteris, M.P. Chronopoulos, C.Z. Chrysostomou, Seismic vulnerability assessment of historical masonry structural systems, Engineering Structures, 62-63(2014) 118-134.

[5] P. Castaldo, M. De Iuliis, Effects of deep excavation on seismic vulnerability of existing reinforced concrete framed structures, Soil Dynamics and Earthquake Engineering, 64(2014) $102-112$.

[6] Zijun Wang, Boming Zhao, Correlations between structural damage and ground motion parameters during the Ms8.0 Wenchuan Earthquake, Soil Dynamics and Earthquake Engineering, 72(2015) 129-137.

[7] Ministry of Construction P.R.China, Code for seismic design of buildings (GBJ11-89), Seismological Press Publication, Beijing, 1990.

[8] Ministry of Construction P.R.China, Code for seismic design of buildings (GB50011-2001), China Construction Press Publication, Beijing, 2002.

[9] Madas P., Elnashai A.S, A new passive confinement model for transient analysis of reinforced concrete structures, Earthquake Engineering and Structural Dynamics, vol. 21 (1992) 409-431.

[10]Crisafulli F.J, Seismic Behaviour of Reinforced Concrete Structures with Masonry Infills, PhD Thesis, University of Canterbury, New Zealand,1997.

[11]Fib., Retrofitting of Concrete Structures by Externally Bonded FRPS, with Emphasis on Seismic Applications. Submitted to FIB Bulletin n. 35, Federation Internationale du Beton, (2006) 220.

[12]Jian Zhu, Ping Tan, Vulnerability analysis of RC factory buildings. Proceedings of Third International Conference on Modelling and Simulation, ICMS2010, IEEE Computer Society Publications. Wuxi, P.R.China, (2010) 47-51.

[13] Casotto, V. Silva, H. Crowley, R. Nascimbene, R. Pinho, Seismic fragility of Italian RC precast industrial, structures, Engineering Structures, 94 (2015) 122-136. 